\title{
PROTOCOL FOR PRE-MANIPULATIVE TESTING OF THE CERVICAL SPINE
}

This document is based on the Australian Manipulative Therapist Association's Protocol for Pre-Manipulative Testing of the Cervical Spine and has been collated by the Manipulative Therapists' Group of the South African Society of Physiotherapy (MTG). Additions to the original text are indicated by asterisk signs.

\section{INTRODUCTION}

MTG is concerned about the safety of manipulation and mobilisation techniques as applied to the cervical spine. Although incidents of trauma to the Vertebral artery after manipulation of the cervical spine by physiotherapists are very rare, even one incident is obviously undesirable.

\section{INCIDENTS AND ACCIDENTS OF CERVICAL MANIPULATION 1947-1986}

The 58 cases of vertebrobasilar complications following cervical manipulation gleaned from a review of the English language literature are briefly summarized.

Complications reported were predominantly following chiropractic manipulation. Of the 54 cases in which details could be determined, manipulation was performed in 44 cases by chiropractors, in 4 cases by osteopaths, and in 2 cases each by medical practitioners, physical therapists, and untrained personnel. The complications were experienced by young adults (mean age 37.3 years, range 7 to 63) who often underwent multiple manipulative procedures at the incident session, not infrequently had warning symptoms or signs of potential vertebrobasilar insufficiency (VBI) prior to the manipulation session (16 cases), and had early onset of neurologic symptoms which progressed to permanent deficit or death. In 45 cases, the neurologic symptoms developed within minutes of manipulative thrust techniques being applied. The most frequent description was of a rotation manipulation. This occurred in 29 out of 32 cases in which the direction was specified. Pre-manipulative dizziness testing was reported in only one case. Grant (1988)

\section{VERTEBROBASILAR INSUFFICIENCY}

Manipulation of the cervical spine is potentially a dangerous procedure because of the spine's intimate relationship with the vertebral artery together with the possibility of congenital anomalies and pathology which cause instability of the upper cervical spine. As a consequence there are several contra-indications to manipulation of the cervical spine.

It is not the intention of this document to present a list of contra-indications to cervical manipulation. Rather, the topic is confined to pre-manipulative testing for possible vertebrobasilar insufficiency (VBI).

Dizziness is commonly the first symptom of VBI and its presence necessitates careful screening before any manipulative procedure is undertaken. However, complications of VBI have arisen following cervical manipulation of patients with no prior VBI symptomatology.

**It is important to be aware of the whole VBI Syndrome.

The Vertebrobasilar vessels are the vessels supplying the brain stem, and occipital lobe and thalamus and even part of the temporal lobe. Symptoms relate to the structures supplied by these vessels. If any branches are compromised, appropriate symptoms result. The groups of structures supplied cause specific symptoms and signs.

i) Occipital lobe: temporary or permanent blindness, homonymous hemianopia or fortification spectra (zigzag lines, spots, stars etc.) may suggest occipital ischaemia via the posterior cerebral arteries.

ii) Cerebellum and vestibular nucleus connections: insilntciency to these areas causes dizziness but may also cause nystaginius, vertigo, ataxia, intention tremor, dysarthric speech or various co-ordination problems without dizziness.

iii) All cranial nerves in the brain stem e.g.

III, IV, VI may cause double vision or pupillary changes

$V$ may cause sensory loss or facial pain

VII may cause facial paralysis

VIII balance or hearing loss

IX \& X swallowing or speech problems

XII tongue movement and articulation problems

iv) Long tract involvement of all fibre tracts conveying information between the cortex and spinal cord i.e. single arm and leg or any combination of unilateral, bilateral or contralateral arm and leg disability in terms of power, reflex, pin prick, light touch, position and vibration sensory loss.

Although dizziness is commonly the presenting symptom of VBI, it must be remembered that it may not be present, whereas other symptoms may well exist and would also contra-indicate manipulation.

It is important to remember that it is not only manipulation which is potentially harmful. Mobilisation techniques, especially at the end of range, can be similarly harmful.**

The procedures outlined in this protocol should ensure that physiotherapists exercise responsibility in using the effective manipulation techniques at their disposal. However, it must be remembered

- that an element of unpredictability remains and incidents do occur even when all pre-manipulative tests are negative and even when the patient has responded favourably to manipulative treatment in the past

- that the test procedures themselves hold certain risks

- that it is important to record carefully and accurately all dizziness tests and pre-manipulative testing procedures undertaken and the responses to them on the part of the patient

- that even when the patient is made aware of the risks attached to a manipulative procedure, that is, informed consent is obtained, the physiotherapist may still remain legally liable if reasonable care, that is the care expected of the average, competent and prudent practitioner, is not employed.

\section{OUTLINE OF PROTOCOL}

\section{Subjective Examination}

All patients with upper quadrant dysfunction should be questioned regarding the presence of dizziness and other symptoms suggestive of vertebrobasilar insufficiency (VBI). Such symptoms include light headedness, strange sensations in the head, dysarthria, diplopia, drop attacks, blackouts, disturbances of vision, tinnitus (see Section II).

\section{Physical Examination}

\section{Patjent Category:}

- Basic pre-manipulative testing is required for patients who do not complain of dizziness and who respond negatively to specific questioning. (see Section IIb)

\section{Choice of techniques and method of application}

- Cervical manipulation is contra-indicated for patients who have dizziness and other symptoms suggestive of VBI and whose 
tests produce or reproduce dizziness and/or associated symptoms.

- Cervical manipulation is contra-indicated for patients who experience dizziness provoked by cervical movement during or after techniques of examination or treatment.

- For patients who present with dizziness and/or other symptoms of possible VBI origin but whose tests are negative

- any technique which provokes dizziness must not be used.

- passive mobilising techniques should be used in initial treatments and their effect over a 24-hour period known before it is even considered that manipulation be used.

- should progression to manipulation be required, a single gentle manipulation may be used providing all the tests described in Section Ila and IIb are negative (see later).

- In any patient requiring cervical manipulation:

- a generalised rotary manipulation of the cervical spine is dangerous and must not be used

- the use of strong axial traction during a manipulation should be avoided as it is likely to increase the risk of injury to the vertebral arteries

- at the first treatment session in which manipulation is used, a single (not multiple) gentle manipulation should be performed

- dizziness testing in the simulated manipulation position should be performed at all subsequent treatments (not just at the initial consultation) in which the physiotherapist wishes to use cervical manipulation.

\section{Recording}

Physiotherapists should always accurately record the dizziness tests undertaken and the patient response to each test. (see addendum for suggested format). ${ }^{*}$ The MTG strongly advises physiotherapists to keep this evidence.**

\section{**Informing the Patient}

The patient should be informed as to the "nature" of the manoeuvre that will be performed.

If the vertebral artery test is positive, the patient should be advised accordingly and warned against any forced manipulative procedure which involves the cervical spine at that stage. This would not exclude manipulation at a later stage should there be no evidence of vertebral artery insufficiency on retesting.**

\section{DETAILS OF THE CLINICAL EVALUATION}

In any patient for whom treatment of the cervical spine is to be undertaken, the presence or development of dizziness, or other symptoms of possible vertebrobasilar insufficiency (VBI) is carefully assessed.

The four stages are:

- subjective examination

- physical examination

- symptoms provoked during treatment of the cervical spine

- symptoms following treatment.

\section{SECTION I: SUBJECTIVE EXAMINATION}

In every patient presenting with upper quadrant dysfunction, questioning is specifically directed to elicit the presence of dizziness. Should the patient suffer from dizziness then questioning proceeds further and must reveal

- the type, degree, frequency and duration of the dizziness, the occurrence, or aggravation of dizziness by head movements and by sustained positions of the head and neck, particularly rotation, extension or combinations of these movements. Any other movement or posture volunteered by the patient is also recorded
- the nature and type of any symptoms associated with the dizziness, for example, light headedness, strange sensations in the head, dysarthria, diplopia, dysphagia, drop attacks, blackouts, disturbances of vision, tinnitus

- the history of dizziness vis a vis the history of the neck, headache or other symptoms

- the status of the dizziness; is it improving, worsening or staying the same?

- previous treatment (if any) and its effect in relieving, exacerbating or producing dizziness and/or associated symptoms.

* Although dizziness is commonly the presenting symptom of VBI, it must be remembered that it may not be present, whereas other symptoms may well exist. Questions must also be asked to elicit presence of other symptoms, which would also contra-indicate manipulation."* (See "VBI Syndrome")

\section{SECTION II: PHYSICAL EXAMINATION}

\section{Ila: Basic Pre-manipulative Scanning of Patients without Dizziness; Standard Routine Tests}

Sitting and/or supine lying (if tests are +ve in sitting, no need to repeat in lying):

- sustained extension

- sustained rotation to the left and right

- sustained rotation with extension, to left and right

- simulated manipulation position. Here the patient's head and neck are held in the manipulation position as a sustained premanipulative procedure.

The patient is questioned regarding dizziness and any other symptoms both during each test and after each test position has been released. The therapist also observes the eyes for nystagmus. Each test position is maintained with over pressure for a minimum of 10 seconds (or less if symptoms are evoked) and upon release, the therapist needs to be aware that latent responses can occur. Recent research suggests that this minimum time may be insufficient to elicit VBI symptoms.

Routine physical examination of the cervical spine will take the patient's neck to the end of the available physiological ranges of extension and of rotation with over pressure applied. However, in patients where these movements are restricted by pain and stiffness, full range cannot be obtained and such compromises as may be placed upon the vertebral arteries may not occur. In such cases a manipulation may not be performed.

\section{lib: Additional Tests to be Undertaken for Patients who Present with Dizziness}

- testing the position or movement which provokes dizziness as described by the patient

- quick movement of the head through the available range of movement when the patient relates dizziness to quick movements of the head rather than head posture or positions

- when dizziness is provoked upon rotation, either during sustained postures or repetitive motion, these tests are further explored in the standing position in order to differentiate dizzjness arising from the vestibular apparatus of the inner ear from that caused by neck movement: head held still, sustained trunk rotation to left and right; or head held still, repetitive trunk rotation to left and right

- sustained positions are adopted for a minimum of 10 seconds or less if symptoms are provoked. These tests when positive, suggest that the patient's dizziness is not caused by inner ear labyrinth disturbance.

\section{lic: Summary}

Cervical manipulation is contra-indicated when any VBI test is positive, that is, a test produces or reproduces dizziness and/or asso- 
ciated symptoms.

Manipulative procedures are contra-indicated if dizziness and/or associated symptoms are provoked whilst adopting a treatment position, during a treatment technique or following a treatment technique. The treatment technique provoking such symptoms is also then contra-indicated. Patients should be warned of the dangers of manipulation of their cervical spine.

** A manipulative technique may be conducted if VBI tests are negative and no contra-indications to cervical manipulation have been elicited on specific overall assessment.**

\section{References}

* Grant R (1988) Dizziness Testing and Manipulation of the Cervical Spine. Clinics of Physical Therapy. Vol 17,7,111-124. Churchill Livingstone. New York.

** The Manipulative Therapists' Group of the South African Society of Physiotherapy.

\section{Acknowledgements}

1. Australian Joumal of Physiotherapy 1988; 34(2):97-100 for permission to use and print extracts from their Protocol.

2. Professor Vivian Fritz, Department of Neurology, University of the Witwatersrand for her advice .

\section{ADDENDUM:}

\section{PRE-MANIPULATIVE DIZZINESS TESTING}

\section{Date: Patient's Name:}

Subjective questioning

dizziness yes no

other possible VBI symptoms yes no

type:

Physical tests

Dizziness with

- sustained extension

- sustained rotation (L)

- sustained rotation (R)

- sustained rot (L)/ext

- sustained rot (R)/ext

- supine, sustained

manipulation position

- other position or

movement nominated

by patient

- quick rotation (L)

- quick rotation (R)

- quick other movements

- standing

head held still, trunk rotation trunk rotation

(L) - labyrinth yes/no

(R) - labyrinth yes/no supine yes no latent

\section{PHYSIOTHERAPISTS WORK IN THE UNITED STATES}

We handle all Licensure and Visa paperwork. Minimum commitment of one year required.

Write or phone collect: THERAPY RESOURCE NETWORK

P O Box 5430355 N Main Street

Plymouth, MI 48170, USA

091 (313) 455-6660

\section{CLASSIFIED ADVERTISEMENTS GEKLASSIFISEERDE ADVERTENSIES}

\section{CAPE TOWN}

Part time (afternoons) physiotherapist required for private practice.

Treatment of respiratory in- and out-patients, cardiothoracic, intens-

ive care, etc.

Starting date 1 March 1991.

CONTACT: Mrs S Pollock (021) 797-1669.

PRETORIA, (OOSTELIK)

PRAKTYK TE KOOP

Gevestigde sentraal geleë praktyk te koop.

KONTAK: (012) 348-9194 (w) (012) of 998-6494 (h).

\section{UNITED STATES}

Physical Therapists wanted to work in a variety of settings, in various geographical locations in the USA.

Minimum of one year commitment.

Will assist with licensurre, working visa and job counselling.

Send resume. Photo desirable.

Include address and ttelephone number (include city code).

SEND TO:

Health Search, 28816 Eight Mile, Suite 105, Livonia, Michigaan

48152, USA.

May call collect (between 10 am and 4 pm, Eastern Standard Time, Monday to Friday) (313) 47103893.

APPARATUS FOR SALE
Bird Mark 8 Respirator
plus various accesories (nebulisers, masks, oxygen regulator, instuc-
tion and various booklets etc.)
Price: R2,800
CONTACT: Mr W P Roets (0391) 74225.

NELSPRUIT

LOCUM REQUIRED

Locum required for private practice from April 1991.

CONTACT: Carol Shrosbree (01311) 44311 (w), 43384 (h).

JOHANNESBURG

Part time physiotherapist required for the new St Giles Home in

Johannesburg, a rehabilitation centre dealing with adults. New gym-

nasium and therapeutic pool.

CONTACT: Anthony Cosgrove (011) 615-7681.

PRETORIA

Equipment for sale

Siemens Interferential with suction unit

Siemens Megatherm Shortwave

TruTrac traction unit

Omron nebuliser

Trolleys, cubicle curtains and other bits and pieces.

CONTACT: Shirley Castleman (012) 380-0081 9 am to $5 \mathrm{pm}$.

\section{JOHANNESBURG}

Physiotherapist required for private practice in Turffontein. Interes-

ting and varied work.

CONTACT: (011) 683-4244 (w), 442-8238 (h).

WERKSVAKANSIE IN ENGELAND

Fisioterapeute en arbeidsterapeute.

KONTAK asseblief: Dr Charl Laubscher, Posbus 12099, Centrahill 6006 (Port Elizabeth).

Telefoon: (041) 42-1061 (w), 55-7764 (h) 